\title{
A rare occurrence of Guillain-Barré syndrome after off-pump coronary artery bypass surgery
}

\author{
Esra Eker ${ }^{1}$, Ali Kemal Gürr ${ }^{2}$, Emrah Şişli ${ }^{2^{\star}}$ \\ ${ }^{1}$ Department of Anesthesiology and Reanimation, Van Education and Research Hospital, Van, Turkey \\ ${ }^{2}$ Department of Cardiovascular Surgery, V an Education and Research Hospital, Van, Turkey
}

\begin{abstract}
Guillain-Barré syndrome (GBS) is an acute inflammatory polyradiculoneuropathy resulting in symmetrical muscle weakness and diminished reflexes. With lack of exact etiology, GBS usually develops as an antecedent infection. Other than the known risk of neurological complications after coronary artery surgery, which includes intracranial hemorrhage, embolic events and transient ischemic attack, we aimed to present a patient in whom GBS developed after an off-pump coronary artery bypass grafting $(\mathrm{CABG})$.

A 56 year-old-male presented muscle weakness and somnolence on the postoperative second day of CABG. There was no pathology other than suspicious plaque formations in cranial magnetic resonance imaging. Nerve conduction velocity study revealed prolonged distal latency, conduction velocity slowing and temporal dispersion of compound action potential which were all indicative of demyelination. Along with the clinical findings and high protein, glucose and cell number detected in cerebrospinal fluid, the diagnosis of GBS was made. The management of $0.4 \mathrm{gr} / \mathrm{kg} / \mathrm{day}$ intravenous immunoglobulin was initiated. During a period of 15 -day intensive care unit follow-up, the symptoms started to improve at the $72^{\text {th }}$ hour of intravenous immunoglobulin therapy. The patient was discharged in good clinical condition on postoperative 42 days.

Despite the pathophysiological mechanism of GBS is still unknown, one should remain alert to the development of GBS after cardiac surgery because, the disease yields favorable prognosis once the diagnosis is made early, and the treatment, either plasmapheresis and/or IVIG, is initiated at once.
\end{abstract}

Key words: Guillain-Barré syndrome, coronary artery bypass, postoperative complications

\section{Introduction}

With the annual incidence of $0.38-2.53 / 100.000$, Guillain-Barré syndrome (GBS) is an acute polyradiculoneuropathy caused by an immune response targeting axon, myelin or both, usually following an antecedent infection (1). While recovery can take anywhere from a few weeks to few years, $90 \%$ of patients with GBS convalescence completely or with mild neurologic deficit within one year. It can be fatal with $1-15 \%$ risk of death when the respiratory drive is compromised $(1,2)$. Regarding the development of GBS after cardiac surgery, the literature cannot go further than case reports (3-7). Thus, we aimed to present a rare incidence of GBS that developed early after off-pump coronary artery bypass grafting $(C A B G)$ and completely subsided within 45 days without neurological deficit.

\section{Case Report}

A 56-year-old male with the complaint of effortrelated chest pain was further evaluated through treadmill test in which, electrocardiographic changes were detected, and chest pain occurred. The patient had no history of autoimmune disease. In transthoracic echocardiography ejection fraction was 55\% without significant valvar pathology. There was no akinesia or dyskinesia. There was no significant stenosis in bilateral carotid Doppler interrogation. The patient was evaluated by coronary angiography, which revealed; $25 \%$ middle right coronary artery, $70 \%$ proximal left anterior descending coronary artery and 90\% second diagonal coronary artery stenosis.

Through median sternotomy, off-pump coronary artery bypass grafting $(C A B G)$ procedure in which left internal mammary artery to left anterior descending coronary artery, and saphenous vein to

\footnotetext{
*Corresponding Author: Emrah Şişli, MD. Department of Cardiovascular Surgery, Van Education and Research Hospital. Süphan district, İpekyolu street, 65400, Edremit, Van 
Eker et al / Guillain-Barré syndrome and coronary surgery

Table 1 . The results of motor nerve conduction velocity study

\begin{tabular}{|c|c|c|c|c|}
\hline Nerve & Latency (msec) & Amplitude (mV) & Distance $(\mathrm{cm})$ & Velocity $(\mathrm{m} / \mathrm{sec})$ \\
\hline \multicolumn{5}{|c|}{ Left median } \\
\hline Wrist & 4.15 & 1.5 & & \\
\hline Elbow & 8.85 & 1.2 & 24 & 51.1 \\
\hline \multicolumn{5}{|c|}{ Left Ulnar } \\
\hline Wrist & 3.35 & 0.8 & & \\
\hline Elbow & 8.15 & 0.8 & 24 & 50.0 \\
\hline \multicolumn{5}{|c|}{ Left tibial } \\
\hline Ankle & 5.95 & 1.2 & & \\
\hline Knee & 18.0 & 1.0 & 44 & 36.5 \\
\hline
\end{tabular}

diagonal branch end-to-side anastomoses were performed. There was no hemodynamic compromise during surgery. The patient was transferred to the intensive care unit (ICU) under stable hemodynamic condition with $5 \mathrm{mcg} / \mathrm{kg} / \mathrm{min}$ dopamine and $3 \mathrm{mcg} / \mathrm{kg} / \mathrm{min}$ nitroglycerin infusion. By three hours of ICU follow-up, the patient was electively extubated though the hemodynamic status was stable, there was no significant mediastinal bleeding, the rhythm was sinus, the arterial blood gas parameters were within normal ranges and the neurologic status of the patient was normal.

With the gradually increasing confusion and ascending muscle weakness on postoperative second day, the patient was consulted with neurology. In neurologic examination, along with confusion, the patient had lost knee jerks. The patient had grade $1 / 5$ (able to move a muscle but no movement of limb, especially inability to overcome gravity) muscle weakness in the lower extremities. The patient was evaluated by cranial computed tomography (CT) and magnetic resonance imaging (MRI). Other than suspicious plaque formations on cranial MRI, there was no pathology detected either on MRI or CT. The muscle weakness ascended and involved the upper extremities in preceding two days. Lumbar puncture for cerebrospinal fluid (CSF) analysis revealed increased protein level $(680 \mathrm{mg} / \mathrm{dl})$ without accompanying pleocytosis. Nerve conduction velocity (NCV) study revealed prolonged distal latency, conduction velocity slowing and temporal dispersion of compound action potential of which were all indicative of demyelination. The measurements of nerve conduction velocity study are presented in (Table 1). As a result, the patient was diagnosed with GBS, and intravenous immune globulin (IVIG) was initiated as four daily infusions for a total dose of $0.4 \mathrm{gr} / \mathrm{kg} /$ day.
The patient was monitored in a critical care setting with attention to vital signs, cardiovascular status and pulmonary physiotherapy. As well as turning and assiduous skin care, daily range-of-motion exercises were initiated. Although the neurological status and paralysis started to decrease in intensity after 72 hours, the patient was followed-up in a critical care setting for a period of 15 days. The patient was discharged with good hemodynamic condition and full functional recovery on postoperative 42 days.

\section{Discussion}

The article of Gensicke et al. (2) had attracted notice in regard to the increasing incidence of GBS after surgery, but none of the six postsurgical patients was operated for cardiac surgery. Being an autoimmune neurological disease, GBS can develop de novo after cardiac surgery in which little is known about the exact mechanism. Cardiopulmonary bypass, which leads to significant inflammatory burden on patient, seems to be a facilitative factor for development of GBS. On the other hand, to the best of our knowledge, the literature included six patients in which GBS had occurred after cardiac surgery (3-7). While five of them were performed under CPB (4-7), the $\mathrm{CABG}$ procedures reported by $\mathrm{Cingoz}$ et al. (3) was performed off-pump, as in our case. This situation may bring to front the genetic factors and exposure to an unknown antigen involved in the pathogenesis of GBS developing after cardiac surgery. Our patient had no history of autoimmune disease. Additionally, it is impossible and not cost-effective to evaluate all patients by means of genetic counselling who are at risk of GBS.

Although the prognosis is usually favorable in the majority, the outcome of patients with GBS is 
worst when severe proximal motor and sensory axonal damage occurs because in axonal damage, successful regeneration cannot occur $(1,2)$. There was no axonal damage in our patient which may be the reason of our patient's quick response to the IVIG therapy.

The case reported by Cingoz et al. (3) resembled to our patient both the onset of symptoms and the extensiveness of the disease. The difference was that while IVIG was the only treatment utilized in our case, they utilized plasmapheresis for 5 days in which the intensification and recovery period was shorter than that of our patient (discharged on postoperative day 10). Plasmapheresis also enabled early ambulation and rehabilitation which in our opinion was an important part of the treatment. Although plasmapheresis is considered the most effective therapy, early administration of IVIG was also shown to shorten the length of paretic intensification of which both therapies were considered the first-line treatment $(1,2)$. Because the neurological status of our patient responded quickly to IVIG treatment, we did not utilize plasmapheresis.

Despite the pathophysiological mechanism of GBS is still unknown, one should remain alert to the development of GBS after cardiac surgery because, the disease yields favorable prognosis once the diagnosis is made early, and the treatment, either plasmapheresis and/or IVIG, is initiated at once.

\section{Conflict of interest}

None declared.

\section{References}

1. McGrogan A, Madle GC, Seaman HE, de Vries CS. The epidemiology of Guillain-Barre syndrome worldwide. A systematic literature review. Neuroepidemiology 2009 ;32: 150-163.

2. Gensicke H, Datta AN, Dill P, Schindler C, Fischer D. Increased incidence of Guillain-Barre syndrome after surgery. Eur J Neurol 2012; 19: 1239-1244.

3. Cingoz F, Tavlasoglu M, Kurkluoglu M, Sahin MA. Guillain-Barre syndrome after coronary artery bypass surgery. Interact Cardiovasc Thorac Surg 2012; 15: 918-919.

4. Hekmat M, Ghaderi H, Foroughi M, Mirjafari SA. Guillain-Barre Syndrome after Coronary Artery Bypass Graft Surgery: a Case Report. Acta Med Iran 2016; 54: 76-78.

5. Hogan JC, Briggs TP, Oldershaw PJ. GuillainBarre syndrome following cardiopulmonary bypass. Int J Cardiol 1992; 35: 427-428.

6. Renlund DG, Hanley DF, Traill TA. GuillainBarre syndrome following coronary artery bypass surgery. Am Heart J 1987; 113: 844-845.

7. Punith K, Sudhir U, K. R, Anil Kumar T. Guillian-Barre syndrome following coronary artery bypass surgery. Indian J Med Spec 2011; 2: 157-159. 